\title{
Estimation method for determining surface film conductance during cooling of fish packages.
}

\begin{abstract}
This paper presents an alternative method for determining the surface film conductance of an infinite fish slab subjected to the cooling process. Many methods have been published, but their solutions have inherent appreciable inaccuracy and limitations. The present authors used the temperature histories of five locations within a slab sample of fish, obtained by the experimental investigation part of this work, along with the inverse heat conduction problem (IHCP) technique to develop a correlation for variable surface film conductance. When the above correlation was used for temperature predictions, the predicted and experimentally measured temperature distribution profiles were compared numerically. Better agreement than that implemented by other investigators was achieved. This revealed the accuracy and superiority of the present method, and the limitations of other methods are overcome in this method.
\end{abstract}

Keyword: Cooling; Fish; Surface Film Conductance; IHCP. 